\title{
An Approach for Developing Uncertain Multi-perspective Expert System Using the Algebraic Sum Method
}

\author{
Musbah J. Aqel \\ Department of Electrical and Computer Engineering, Faculty of Engineering \\ Applied Science University, Amman-11931, Jordan
}

\begin{abstract}
A multi-perspective expert system approach based on algebraic sum method was developed to deal with uncertain knowledge and provides different perspectives for different experts while dealing with single knowledge base according to experts' interests. However, assertion value for each perspective is calculated and by using the algebraic summation method a conclusion can be determined.
\end{abstract}

Key words: Multi-perspective expert system, medical expert systems, algebraic sum method, bayes theorem, uncertainty

\section{INTRODUCTION}

Different interpretation has been given to the notion of perspectives. The most interested one is the multi-perspective experts' one. A perspective of a world is the perception an expert of a particular discipline has of this world. Different experts watching an object will observe different attributes according to their interests. Nevertheless, if two experts watch the same attributes of a same object, then they will see the same value ${ }^{[1]}$. Experts interested in the same concept (knowledge base) will propose different partitions of this world and will consider a different set of attributes for the individuals of the concept; for example, animals may be classified according to different criteria: anatomy, evolution, habitat, etc. This notion has been implemented in some medical expert systems ${ }^{[2,3]}$.

Instead of developing multi expert systems to satisfy a multi expert interest (i.e. perspective) this can be implemented in one expert system with one knowledge based that can satisfy a multi experts' perspectives.

Much of information that is used in some expert systems especially medical type is probabilistic and uncertain. So, many expert systems are developed to deal with such type of knowledge using Bayes' theorem. In order to be able to apply the Bayes theorem, there are two basic assumptions must be satisfied. First, the diseases under consideration should be mutually exclusive and exhaustive. Second, disease manifestations should be independent $t^{[4]}$. Due to these limitations, algebraic summation method can better used to solve these problems ${ }^{[5]}$.

In this paper, an approach for a multi-perspective expert system is proposed and developed that can deal with probabilistic and uncertain knowledge base using the algebraic sum method.
An approach for uncertain multi-perspective expert system: The approach for uncertain multi-perspective expert system can be described as follows:

Knowledge base concepts: The knowledge base usually consists of a group of independent concepts, which sets up an initial partition of the limited world model. Concepts are independent and each concept consists of a set of perspectives. Each concept is represented by a set of classes, predicates, rules, etc. The individuals of a concept may be viewed from different perspectives, one for each expert's perspective.

Knowledge coding: The information in the knowledge base by including a coding number for each fact in the knowledge base. This code consists of three parts:

$<$ I. Pi. Di $>$

Where

I : is a selected integer number that represents the information in the knowledge base.

$\mathrm{Pi}$ : represents the inclusion of this information on the perspective I.

Di: represents the assertion value for this information in the current perspective. This value is calculated as a fractional value. Where a positive value of $\mathrm{Di}$ represents a supporting and assertion of this value, while a negative value represents denying of this value from the current perspective.

For example: suppose the following fact has the following code:

Sputum gram stain streptococcus: Assume current perspective is 2 and assertion value for this fact is 0.035 . Then the code will be formed as follows: 322.2.035.

Corresponding Author: Musbah J. Aqel, Department of Electrical and Computer Engineering, Faculty of Engineering, pplied Science University, Amman-11931, Jordan 
Table formation and threshold: The information, which was input and represented by the coding process, will be arranged in a table according to the importance of the query for only current perspective that should be pre-determined at the time of query. After calculating the total sum of $\mathrm{Di} \mathrm{x} \mathrm{Pi}$, then, if the sum is greater than a threshold value, then, an inference can be obtained.

Approach processing: During the process of the approach, the query will be assisted and whenever there is a match between the entered query information and that available at the current perspective in the knowledge base, the integral part of a relevant data can be separated by using a function to obtain the assertion number where a negative value for a data will be used as an absolute value. These asserted values, will be calculated as algebraic sum. This sum then will be a multiplied by 1000 to transform it into integer, then the perspective number will again be multiplied by a perspective weight value that assigned for each perspective as follows:

$\mathrm{P} 1=10, \mathrm{P} 2=100, \ldots . \mathrm{Pi}=10 \mathrm{i}$.

This will insure that the same fact (piece of information) that is available in the knowledge base can be used in different perspectives but with different values.

Approach implementation and example: An expert system for the upper respiratory system diseases has been developed earlier with a multi perspective notion but it was based on exact knowledge. Here, the same expert system is modified with a probabilistic knowledge is included while developing this medical expert system. While developing this system, a computing diagnostic value sum subprogram was developed. This program calculates the diagnostic value sum for the various diagnostic entities of a certain disease group. Then another subprogram compares the diagnostic value sum with the diagnostic threshold to prompt a diagnosis. The diagnostic certainty is based on the coincidence degree of the diagnostic value sum with the diagnostic threshold.

The expert system knowledge base consists of the following perspectives:

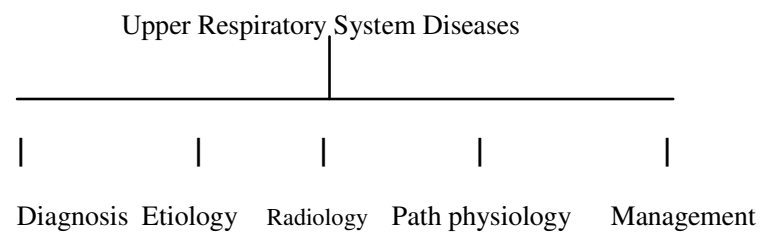

These five perspectives concern different specialist in different fields of medicine and each one has his/her own interest in the same concept (i.e. upper respiratory system diseases). For example, if a patient with Pneumonia is coming to a clinic and consulted an internal medicine specialist, then the following fact is asserted as follows:

The patient's sputum gram stain has shown streptococcus pneumonia organism.

This fact is a concern of the following specialists:

Internal medicine: to help in the manifestation and diagnosis process.

Etiologist: to know that this type of organism causes Pneumonia.

Radiologist: To match the infiltrate pattern at the X-ray since each organism has different pattern of infiltrate.

Medical management staff: The choice of antibiotic treatment is based on the most likely etiology organism.

So, this fact will be having an assertion value and will participate in each perspective differently to produce a conclusion according to the above approach.

Suppose that the internal medicine specialist would like to use this system, then according to this system, the user is concerned in the first perspective. The following symptoms are coded in the system partially as follows:

400 - Breathlessness

421 - Bloody stool

610 - History of upper respiratory infection

1180 - Eosinphillia

444 - Streptococcus pneumonia organism

384 - Thrombocytopenia

501 - Pleural pain

2229 - Abdominal pain

799 - Tachycardia

832 - Diarrhea

330 - Haemothysis

The value of $P$ (perspective) is equal to (1), the value of $\mathrm{D}$ (assertion value) could be given in the table(1) for coded information for pulmonary edema as follows:

Table(1) Diagnostic data set of encoded information

\begin{tabular}{|l|l|l|l|}
\hline$<400,1,0.1>$ & $<501,1,0.1>$ & $\begin{array}{l}<421,1,- \\
0.1\end{array}$ & $\begin{array}{l}<384,1,- \\
0.2\end{array}$ \\
\hline$<444,1,0.1>$ & $<501,1,-0.1$ & $<330,1,-$ & $<799,1,0.2$ \\
& & 0.2 & \\
\hline$<1180,1,0.0 .01$ & $<610,1,0.01$ & $<2229,1,-$ & $<832,1,-$ \\
& & 0.2 & 0.1 \\
\hline
\end{tabular}

For example, from the table the data set $<799,1,0.2>$ shows the coding value for tachycardia in the internal perspective, and the positive value shows the manifestation for diagnosis of this symptom in any given disease (i.e. Pulmonary edema) at this case. 
While the value $<832,1,-0.1\rangle$, negative value means that the patient information is expressed to deny this symptom (diarrhea) for this case.

\section{CONCLUSION}

An approach for probabilistic multi perspective expert system development using algebraic sum method is proposed where the importance of information to the current query called assertion value can be evaluated and the algebraic sum for these asserted values should be calculated to make a decision in a given perspective. However, instead of developing an expert system for each expert's perspective, a multi perspective approach can suffice this goal. Moreover, since the medical knowledge, which is required to make a decision is probabilistic. So, the proposed approach could provide this facility, where this approach is based in algebraic method.

\section{REFERENCES}

1. Musbah, J.A., 2000. An approach for a multiperspective expert systems development. J. AMSE, Advances in Modeling and Analysis, 43: 3-4

2. Musbah, J.A. and M. Eweissi, 1998. Multi perspective expert system for children emergencies. J. AMSE, Modeling-Measurement and Control, 18: 1-2.

3. Musbah, J.A., 2000. A cooperative decisionmaking multi perspective expert system for respiratory system diseases. J. AMSE, Advances in Modeling and Control, 45: 3-4.

4. Chenglin Peng, et al., 1996. Applying Bayes Theorem in Medical expert systems. IEEE Eng. Med. and Biol.

5. Xiao, S.Z. and C. Tingfu, 1985. Algebraic sum method in biomedical computer application system. Microelectronics \& Computer, 2: 11-15. 